International Journal of Difference Equations (IJDE).

ISSN 0973-6069, Volume 16, Number 1, (2021). 11-17

(C) Research India Publications

https://dx.doi.org/10.37622/IJDE/16.1.2021.11-17

\title{
Laplace Transform of Discriminant for Functions of Second Variable and its Generalized Form
}

\author{
Hwajoon Kim
}

Kyungdong University, Yangju, S. Korea.

\begin{abstract}
We consider the Laplace transform of discriminant for functions of second variable and its generalized form. The generalized form is considered to be extended to the case of noninteger order. This method can be applied well to heat equations or other partial differential equations.
\end{abstract}

AMS Subject Classification: 44A05, 35K05, 35A22

Key Words and Phrases: discriminant, integral transform, noninteger order, PDEs

\section{INTRODUCTION}

Interpretation of partial differential equations(PDEs) using integral transforms have been tried in various ways. Since almost all the existing integral transforms[1-11] can be transformed by the Laplace transform in essence, it is known that the transform is the easiest to apply to the solution of PDEs. The discriminant for functions of second variable is given by

$$
D=\left|\begin{array}{cc}
f_{x x} & f_{x y} \\
f_{y x} & f_{y y}
\end{array}\right|=f_{x x} f_{y y}-f_{x y}^{2}
$$

where

$$
f_{x x}=\frac{\partial^{2} f}{\partial x^{2}} .
$$

Since the discriminant $D$ is integer-order, its Laplace transform representation is not difficult. The proposed method can be usefully applied when the initial value or 
boundary value is uncertain. This is because arbitrary constants included in the solution of PDEs can be expressed in some detail as initial values. Let us extend the representation to the case of noninteger order. We recall that the Laplace transform of the derivatives $f^{(q)}$ of any integer is

$$
£\left(f^{(q)}\right)=s^{q} £(f)-s^{q-1} f(0)-s^{q-2} f^{\prime}(0)-\cdots-f^{(q-1)}(0),
$$

where $q$ is an integer. Converting this equation to a simple form, we have

$$
£\left(\frac{d^{q} f}{d x^{q}}\right)=s^{q} £(f)-\sum_{k=0}^{q-1} s^{q-1-k} \frac{d^{k} f}{d x^{k}}(0), \quad q=1,2,3, \cdots
$$

Even if the positions of $k$ and $q-1-k$ are swapped in this equation, the equation does not matter. Therefore, we have

$$
£\left(\frac{d^{q} f}{d x^{q}}\right)=s^{q} £(f)-\sum_{k=0}^{q-1} s^{k} \frac{d^{q-1-k} f}{d x^{q-1-k}}(0), \quad q=0, \pm 1, \pm 2, \cdots
$$

because

$$
£\left(\frac{d^{q} f}{d x^{q}}\right)=s^{q} £(f), \quad q=0,-1,-2, \cdots
$$

Of course, (2) can be extended to noninteger $q$ by

$$
£\left(\frac{d^{q} f}{d x^{q}}\right)=s^{q} £(f)-\sum_{k=0}^{n-1} s^{k} \frac{d^{q-1-k} f}{d x^{q-1-k}}(0), \text { all } q,
$$

where $n$ is the integer such that $n-1<q \leq n$. If $q<0$, the proof is made using the Riemann-Liouville integral

$$
\frac{d^{q} f}{d(x-a)^{q}}=\frac{1}{\Gamma(-q)} \int_{a}^{x}[x-y]^{-q-1} f(y) d y, q<0
$$

and if $q>0$, it is made using

$$
\frac{d^{q} f}{d x^{q}}=\frac{d^{n}}{d x^{n}} \frac{d^{q-n} f}{d x^{q-n}}
$$

The details are shown in [12].

This article has covered the expressions (1) and (3) of the discriminant $D$ for functions of second variable. 


\section{LAPLACE TRANSFORM OF DISCRIMINANT FOR FUNCTIONS OF SECOND VARIABLE AND ITS GENERALIZED FORM}

Lemma 1. In (2), consider the proof when $q$ is a natural number, i.e.,

$$
£\left(\frac{d^{q} f}{d x^{q}}\right)=s^{q} £(f)-\sum_{k=0}^{q-1} s^{k} \frac{d^{q-1-k} f}{d x^{q-1-k}}(0), \quad q=1,2,3 \cdots
$$

Proof. First let us consider the case of $q=1$.

$$
£\left(\frac{d f}{d x}\right)=s £(f)-f(0),
$$

and by the direct calculation

$$
\begin{gathered}
£\left(\frac{d f}{d x}\right)=\int_{0}^{\infty} e^{-s x} \frac{d f}{d x} d x \\
\left.=e^{-s x} f(x)\right]_{0}^{\infty}+s \int_{0}^{\infty} e^{-s x} f(x) d x=s £(f)-f(0) .
\end{gathered}
$$

Next, let us assume that $q=m$ is valid for an arbitrary natural number $m$. Thus,

$$
£\left(\frac{d^{m} f}{d x^{m}}\right)=s^{m} £(f)-\sum_{k=0}^{m-1} s^{k} \frac{d^{m-1-k}}{d x^{m-1-k}} f(0)
$$

holds. It is sufficient to show that it is

$$
£\left(\frac{d^{m+1} f}{d x^{m+1}}\right)=s^{m+1} £(f)-\sum_{k=0}^{m} s^{k} \frac{d^{m-k}}{d x^{m-k}} f(0) .
$$

Since

$$
£\left(\frac{d^{m+1} f}{d x^{m+1}}\right)=£\left[\frac{d}{d x}\left(\frac{d^{m} f}{d x^{m}}\right)\right]=s £\left(f^{(m)}\right)-f^{(m)}(0)
$$

for $f^{(m)}$ is the $m$-th derivative of $f$, we get

$$
\begin{gathered}
£\left(\frac{d^{m+1} f}{d x^{m+1}}\right) \\
=s^{m+1} £(f)-s \sum_{k=0}^{m-1} s^{k} \frac{d^{m-1-k}}{d x^{m-1-k}} f(0)-f^{(m)}(0) \\
=s^{m+1} £(f)-\sum_{k=0}^{m} s^{k} \frac{d^{m-k}}{d x^{m-k}} f(0)
\end{gathered}
$$

because

$$
s \sum_{k=0}^{m-1} s^{k} \frac{d^{m-1-k}}{d x^{m-1-k}} f(0)
$$




$$
=\sum_{k=0}^{m} s^{k} \frac{d^{m-k}}{d x^{m-k}} f(0)-\frac{d^{m} f}{d x^{m}} f(0) .
$$

Verification of this equality can be easily verified by substitution. Hence, the theorem is valid in an arbitrary natural number $k$.

We would like to consider the Laplace transform of discriminant for functions of second variable.

Theorem 2. The Laplace transform of discriminant of second partial derivative test can be expressed as

$$
\begin{gathered}
\left(s^{2} F^{2}-s f(x, 0) F-f_{y}(x, 0) F, \quad-s^{2} F^{2}, 2 s f_{x}(x, 0) F, \quad-\left(f_{x}(x, 0)\right)^{2}\right) \\
\times\left(\begin{array}{c}
\frac{\partial^{2}}{\partial x^{2}} \\
\left(\frac{\partial}{\partial x}\right)^{2} \\
\frac{\partial}{\partial x} \\
1
\end{array}\right),
\end{gathered}
$$

where

$$
D=\left|\begin{array}{cc}
f_{x x} & f_{x y} \\
f_{y x} & f_{y y}
\end{array}\right|=f_{x x} f_{y y}-f_{x y}^{2} .
$$

Alternately, $£(D)$ can be expressed as

$$
\begin{gathered}
£(D)=\left(s^{2} F^{2}-s f(x, 0) F-f_{y}(x, 0) F\right) \frac{\partial^{2}}{\partial x^{2}}-s^{2} F^{2}\left(\frac{\partial}{\partial x}\right)^{2} \\
+2 s f_{x}(x, 0) F \frac{\partial}{\partial x}-\left(f_{x}(x, 0)\right)^{2} .
\end{gathered}
$$

The generalization of this result to non-integer order is as

$$
\begin{gathered}
£\left(D^{q}\right)=\left(s^{2 q} F-\sum_{k=0}^{n-1} s^{k} \frac{d^{2 q-1-k} f}{d x^{2 q-1-k}}(0)\right)\left(s^{2 q} F-\sum_{k=0}^{n-1} s^{k} \frac{d^{2 q-1-k} f}{d y^{2 q-1-k}}(0)\right) \\
-\left(s^{q} F-\sum_{k=0}^{n-1 / 2} s^{k} \frac{d^{q-1-k} f}{d x^{q-1-k}}(0)\right)\left(s^{q} F-\sum_{k=0}^{n-1 / 2} s^{k} \frac{d^{q-1-k} f}{d y^{q-1-k}}(0)\right),
\end{gathered}
$$

where $n$ is the integer such that $n-1<2 q \leq n$.

Proof. First, let us take Laplace transform for each terms with respect to $y$. Then

$$
£\left(f_{y y}\right)=s^{2} F-s f(x, 0)-f_{y}(x, 0)
$$




$$
\begin{aligned}
& £\left(f_{x x}\right)=\int_{0}^{\infty} e^{-s y} \frac{\partial^{2} f}{\partial x^{2}} d y=\frac{\partial^{2}}{\partial x^{2}} £(f)=\frac{\partial^{2}}{\partial x^{2}} F \\
& £\left(f_{x y}\right)=s £\left(f_{x}\right)-f_{x}(x, 0)=s \frac{\partial}{\partial x} F-f_{x}(x, 0)
\end{aligned}
$$

for $F=£(f)$. Thus,

Thus, the coefficients of

$$
\begin{aligned}
& £(D)=\frac{\partial^{2}}{\partial x^{2}} F \cdot\left(s^{2} F-s f(x, 0)-f_{y}(x, 0)\right)-\left(s \frac{\partial}{\partial x} F-f_{x}(x, 0)\right)^{2} \\
&=\left(s^{2} F^{2}-\right.\left.s f(x, 0) F-f_{y}(x, 0) F\right) \frac{\partial^{2}}{\partial x^{2}}-s^{2} F^{2}\left(\frac{\partial}{\partial x}\right)^{2} \\
&+2 s f_{x}(x, 0) F \frac{\partial}{\partial x}-\left(f_{x}(x, 0)\right)^{2} .
\end{aligned}
$$

and 1 are

$$
\frac{\partial^{2}}{\partial x^{2}},\left(\frac{\partial}{\partial x}\right)^{2}, \frac{\partial}{\partial x}
$$

$$
s^{2} F^{2}-s f(x, 0) F-f_{y}(x, 0) F,-s^{2} F^{2}, 2 s f_{x}(x, 0) F,
$$

and $-\left(f_{x}(x, 0)\right)^{2}$, respectively. We have dealt with the Laplace transform of the integer-order derivative. Consider generalizing this as a noninteger-order derivative. We consider

all $q$. Then

$$
D^{q}=\frac{d^{2 q} f}{d x^{2 q}} \cdot \frac{d^{2 q} f}{d y^{2 q}}-\frac{d^{q} f}{d x^{q}} \cdot \frac{d^{q} f}{d y^{q}}
$$

$$
\begin{gathered}
£\left(D^{q}\right)=\left(s^{2 q} F-\sum_{k=0}^{n-1} s^{k} \frac{d^{2 q-1-k} f}{d x^{2 q-1-k}}(0)\right)\left(s^{2 q} F-\sum_{k=0}^{n-1} s^{k} \frac{d^{2 q-1-k} f}{d y^{2 q-1-k}}(0)\right) \\
-\left(s^{q} F-\sum_{k=0}^{n-1 / 2} s^{k} \frac{d^{q-1-k} f}{d x^{q-1-k}}(0)\right)\left(s^{q} F-\sum_{k=0}^{n-1 / 2} s^{k} \frac{d^{q-1-k} f}{d y^{q-1-k}}(0)\right),
\end{gathered}
$$

where $n$ is the integer such that $n-1<2 q \leq n$. If $n$ is odd, there is no problem. If $n$ is even, we can change $\frac{n-1}{2}$ to $\frac{n}{2}$.

Note that

$$
\frac{\partial^{2}}{\partial x^{2}} \neq\left(\frac{\partial}{\partial x}\right)^{2}
$$

in the above equation. For example, we consider $f(x, y)=x^{2}-y$. Then

$$
\frac{\partial^{2} f}{\partial x^{2}}=2, \text { but }\left(\frac{\partial f}{\partial x}\right)^{2}=4 x^{2} .
$$

Conflict of interest The authors declare no conflicts of interest.

Acknowledgements This research was supported by Kyungdong University Research Fund, 2021. 


\section{REFERENCES}

[1] F. B. M. Belgacem and R.Silambarasan, Theory of natural transform, Mathematics in Engineering, Science and Aerospace, 3 (2012), 105-135.

[2] Bertrand, J., Bertrand, P., and Ovarlez J.P., The Mellin Transform, The Transforms and Applications, Handbook (A.D. Poularkas, ed.). CRC Press, Boca Raton, FL, 1996.

[3] T. M. Elzaki, S. M. Ezaki and E. M. A. Hilal, ELzaki and Sumudu Transform for Solving some Differential Equations, Glob. J. of Pure \& Appl. Math., 8 (2012), 167-173.

[4] Y. H. Geum, A.K. Rathie, and Hj. Kim, Matrix Expression of Convolution and Its Generalized Continuous Form, Symmetry 2020 (2020), 12, 1791.

[5] Jy. Jang and $\mathrm{Hj}$. Kim, An application of monotone convergence theorem in PDEs and Fourier analysis, Far East J. Math. Sci., 98 (2015), 665-669.

[6] Jhanthanam S, Nonlaopon K, Orankitjaroen S. Generalized Solutions of the Third-Order Cauchy-Euler Equation in the Space of Right-Sided Distributions via Laplace Transform, Mathematics, 7 (2019), 376.

[7] $\mathrm{Hj} . \mathrm{Kim}$, The intrinsic structure and properties of Laplace-typed integral transforms, Mathematical Problem in Engineering, 2017 (2017), 1-8.

[8] $\mathrm{Hj}$. Kim, The solution of the heat equation without boundary conditions, Dynamic Systems and Applications, 27 (2018), 653-662.

[9] Wolfram Koepf, Insuk Kim, and Arjun K. Rathie, On a New Class of Laplace-Type Integrals Involving Generalized Hypergeometric Functions, Axioms, 8, (2019), 1-21.

[10] E. Kreyszig, Advanced Engineering Mathematics, Wiley, Singapore, 2013.

[11] Noeiaghdam, S., Fariborzi Araghi, M.A., and Abbasbandy, S. Finding optimal convergence control parameter in the homotopy analysis method to solve integral equations based on the stochastic arithmetic. Numerical Algorithms 81, (2019). https://doi.org/10.1007/s11075-018-0546-7

[12] K. B. Oldham and J. Spanier, The Fractional Calculus, Dover Publications, Inc., New York, 1974.

[13] S. Supaknaree, K. Nonlaopon, and Hj. Kim, Further properties of Laplace-type integral transforms, Dynamic Systems and Applications, 28 (2019), 195-215.

[14] Taejun Sung, Insuk Kim, and Arjun K. Rathie, On a new class of Eulerian's type integrals involving generalized hypergeometric functions, The Australian Journal of Mathematical Analysis and Applications 16, (2019), 1-15. 
[15] Zhaoxuan Wang, Zhiyu Yu, Changlin Tian, Cruz-Rodriguez, Sanchez Batista, and Bin Zhao, Differential equation analysis on COVID-19, Auctores Publishing 2, (2020), 006

[16] G. K. Watugula, Sumudu Transform: a new integral transform to solve differential equations and control engineering problems, Integrated Education, 24 (1993), $35-43$. 
